\title{
O Papel do Hormônio de Crescimento no Tratamento dos Distúrbios Endócrino-Metabólicos do Paciente com a Síndrome da Imunodeficiência Adquirida (Aids)
}

revisão

\author{
angela Maria Spinola-Castro \\ AdRIANA A. SIVIERO-MIACHON \\ Marcos Tadeu Nolasco da Silva \\ GIL GUerRa-Junior
}

Serviço de Endocrinologia

Pediátrica do Departamento de

Pediatria da Universidade

Federal de São Paulo

(Unifesp/EPM), SP, Brasil

(AMSC,AASM);

Serviço de Imunologia

Pediátrica do Departamento de

Pediatria da Faculdade de

Ciências Médicas (FCM) da

Universidade Estadual de

Campinas (Unicamp), SP, Brasil

(MTNS); Serviço de

Endocrinologia Pediátrica do

Departamento de Pediatria da

FCM-Unicamp, Campinas, SP,

Brasil (GGJ).

Recebido em 13/5/2008

Aceito em 22/5/2008

\author{
RESUMO
}

As primeiras descrições da síndrome da imunodeficiência adquirida (Aids) associavam-se à síndrome de emaciamento, e os distúrbios metabólicos às alterações na composição corporal. Após a introdução da terapia anti-retroviral altamente ativa (HAART), houve declínio na desnutrição, e surge a lipodistrofia como importante distúrbio metabólico. A Aids também se caracteriza por distúrbios hormonais, principalmente no eixo hormônio de crescimento/ fator de crescimento insulina-like tipo 1 (GH/IGF-1). O uso do GH recombinante humano ( $\mathrm{hrGH}$ ) foi inicialmente indicado na síndrome de emaciamento, a fim de aumentar a massa muscular. Embora também não existam dúvidas quanto aos efeitos do hrGH na lipodistrofia, a diminuição na sensibilidade à insulina limita o seu uso, o qual ainda não está oficialmente aprovado. A diversidade nos esquemas de tratamento é outro limitante do uso dessa medicação em pacientes com Aids. Esta revisão apresenta os principais distúrbios endócrino-metabólicos associados à Aids e ao uso do $\mathrm{hrGH}$ nessas condições. (Arq Bras Endocrinol Metab 2008; 52/5:818-832)

Descritores: Hormônio do crescimento; Síndrome de imunodeficiência adquirida; Síndrome de emaciação por infecção pelo HIV; Síndrome de lipodistrofia associada ao HIV; Crescimento; Fator de crescimento insulin-like I

\section{ABSTRACT}

The Use of Growth Hormone to Treat Endocrine-Metabolic Disturbances in Acquired Immunodeficiency Syndrome (Aids) Patients.

Acquired Immunodeficiency Syndrome (Aids) was initially related to HIV-associated wasting syndrome, and its metabolic disturbances to altered body composition. After Highly Active Antiretroviral Therapy (HAART) was started, malnutrition has declined and HIV-associated lipodystrophy syndrome has emerged as an important metabolic disorder. Aids is also characterized by hormonal disturbances, principally in growth hormone/insulin-like growth factor 1 (GH/IGF-1) axis. The use of recombinant human $\mathrm{GH}(\mathrm{hrGH})$ was formerly indicated to treat wasting syndrome, in order to increase lean body mass. Even though the use of hrGH in lipodystrophy syndrome has been considered, the decrease in insulin sensitivity is a limitation for its use, which has not been officially approved yet. Diversity in therapeutic regimen is another limitation to its use in Aids patients. The present study has reviewed the main HIV-related endocrine-metabolic disorders as well as the use of hrGH in such conditions. (Arq Bras Endocrinol Metab 2008; 52/5:818-832)

Keywords: Growth hormone; Acquired immunodeficiency syndrome; HIV wasting syndrome; HIV-associated lipodystrophy syndrome; Growth; Insulinlike growth factor $I$ 


\section{INTRODUÇÃO}

A INFECÇÃO PELO VÍRUS da imunodeficiência humana (HIV) e a síndrome da imunodeficiência adquirida (Aids) ainda são considerados problemas de saúde pública, apesar de todos os avanços no diagnóstico e na terapia. Dados da Organização Mundial da Saúde (OMS) permitem estimar que, no final de 2007, havia no mundo 33,2 milhões de pessoas infectadas pelo HIV, sendo 2,5 milhões de casos novos, com 2,1 milhões de mortes atribuíveis à Aids (1). Segundo dados do Ministério da Saúde, até junho de 2007 foram identificados 474.273 casos no Brasil (16.455 em menores de 13 anos), com 32.628 casos novos (2). Entretanto, apesar do aumento no número de casos da doença, o progresso na terapia anti-retroviral tem permitido aumento na sobrevida desses pacientes, de tal forma que, atualmente, há preocupação com a qualidade de vida e com os efeitos ditos secundários ou adversos do tratamento. Existem muitas seqüelas causadas pela doença em suas diferentes fases. Ocorrem alterações imunológicas, nutricionais e endócrino-metabólicas significativas que, em função da sobrevida e do tratamento, também têm mudado de perfil, transformando a Aids atualmente em uma doença crônica, porém ainda incurável (3).

Mudaram também as preocupações em relação ao emprego das terapias alternativas, como é o caso do hormônio do crescimento recombinante humano (hrGH), que tem sido utilizado em pacientes com Aids com diferentes objetivos (4). Inicialmente, foi utilizado para minimizar os distúrbios nutricionais, além de melhorar o crescimento linear e reverter o intenso catabolismo (caquexia), claramente relacionado à sobrevida (5-8). Entretanto, atualmente, sua indicação tem como novo objetivo corrigir a lipodistrofia causada pelas drogas anti-retrovirais (9-11). Adicionalmente, pesquisas recentes atribuem ao hormônio de crescimento $(\mathrm{GH})$ papel significativo no sistema imunológico, como importante regulador da produção de células T (12). Problemas como crescimento, ganho de massa muscular, diminuição da gordura visceral e dos fatores de risco para doença cardiovascular, assim como a melhora da qualidade de vida, tornaram-se importantes. À semelhança do que ocorre com outras doenças crônicas, entre as quais, fibrose cística, doença de Chron, artrite reumatóide e insuficiência renal crônica, os resultados, embora positivos, são objeto de várias controvérsias, especialmente em relação ao tempo de uso, dose e efeitos adversos $(9,10,13,14)$.
O objetivo deste artigo é fazer uma revisão a respeito dos distúrbios nutricionais e endócrino-metabólicos relacionados à Aids e à sua terapêutica, além das principais indicações do uso de hrGH nesses pacientes.

\section{DEFINIÇÕES DE CASO}

Na literatura médica ou na imprensa leiga observa-se, com freqüência, o uso dos termos "soropositivo" ou "portador assintomático" para designar indivíduos infectados pelo HIV sem sintomas clínicos ou imunodepressão. Uma abordagem mais sistemática desta nomenclatura deve enfocar duas situações principais (15):

\section{Indivíduos infectados pelo HIV}

Infecção comprovada laboratorialmente, sem manifestações clínicas graves ou imunodepressão.

\section{Casos de Aids}

\section{Adolescentes $\geq 13$ anos ou adultos}

Infecção pelo HIV comprovada laboratorialmente, associada à doença indicativa de Aids e/ou contagem de linfócitos T CD4 ${ }^{+}$abaixo de 350 células $/ \mathrm{mm}^{3}$.

\section{Crianças ou adolescentes $<13$ anos}

Infecção pelo HIV comprovada laboratorialmente, associada a pelo menos duas doenças indicativas de Aids de caráter leve, ou uma doença indicativa de Aids de caráter moderado ou grave, e/ou contagem de linfócitos $\mathrm{T} \mathrm{CD}^{+}$menor do que o esperado para a idade.

As doenças indicativas de Aids compreendem principalmente as infecções oportunistas, neoplasias e doenças auto-imunes associadas ao HIV (15).

\section{DISTÚRBIOS NUTRICIONAIS E ENDÓCRINO-METABÓLICOS ASSOCIADOS À INFECÇÃO PELO HIV OU AO TRATAMENTO ANTI-RETROVIRAL}

\section{A linha do tempo da história da infecção pelo HIV}

$\mathrm{Na}$ década e meia que sucedeu às primeiras descrições da Aids, a disponibilidade de agentes anti-retrovirais era limitada praticamente à monoterapia, com agentes inibidores da enzima transcriptase reversa, do tipo análogo de nucleosídeo (ITRN), a zidovudina (AZT) e a didanosina (DDI), sendo a eficácia da terapêutica mui- 
to baixa. As complicações infecciosas e as neoplasias decorrentes da infecção pelo HIV eram controladas por medicamentos específicos, que não interferiam com a história natural da doença. Nessa fase histórica da epidemia, a principal conseqüência nutricional e metabólica da infecção, pelas freqüência e gravidade, foi a desnutrição protéico-calórica $(16,17)$. O quadro de desnutrição especificamente associado à infecção pelo HIV, característico desse momento da doença, foi chamado síndrome do emaciamento (ou wasting), e será discutida mais adiante (18).

\section{Mudanças ocorridas com a introdução da terapia anti-retroviral altamente ativa (HAART)}

O ano de 1996 constitui-se em marco na história do tratamento da Aids, com o início do emprego, na prática clínica, de esquemas anti-retrovirais efetivos, constituindo a chamada terapia anti-retroviral altamente ativa ou Highly active antiretroviral therapy (HAART). O esquema terapêutico básico dessa abordagem consiste do emprego de uma terapia combinada com o uso de dois medicamentos do tipo ITRN e um inibidor da enzima protease (IP), ou um inibidor da mesma transcriptase reversa, mas do tipo não análogo de nucleosídeo (ITRNN) (19). Recentemente, novos medicamentos foram introduzidos, dos grupos dos inibidores de fusão, inibidores de integrase e de entrada do HIV (11).

Em decorrência dos avanços na terapêutica, a Aids assumiu o perfil de doença crônica, com possibilidade de controles virológico, imunológico e clínico, embora ainda incurável. Os efeitos sobre a sobrevida, a incidência de infecções oportunistas e a melhora na qualidade de vida de adultos e crianças tratados com os novos esquemas foram dramáticos, estando bem documentados em estudos longitudinais e transversais (17,20-23).

Estudos realizados na fase anterior à introdução da HAART estimam prevalência da síndrome de emaciamento da ordem de 59\% a 84\% (24). A partir de 1996, com a introdução da HAART, tal prevalência reduziuse acentuadamente, se utilizados os critérios do Centers for Disease Control (CDC) (25). Em um estudo de coorte com casuística significativa, já na era HAART, utilizando-se os critérios menos rigorosos propostos pela Conferência de Consenso 2004 (18), documentou-se a presença de ao menos um dos critérios propostos pelo Consenso de 2004 (18) em 38\% dos pacientes (26).
Apesar de o entusiasmo gerado pela eficácia com o controle da replicação viral, começaram a ser identificados efeitos colaterais associados à HAART, dos quais serão destacados aqueles associados ao metabolismo lipídico e de carboidratos. O principal distúrbio metabólico característico dessa fase histórica do tratamento da doença foi a síndrome lipodistrófica $(3,27)$.

\section{Síndrome do emaciamento (wasting) associada ao HIV}

$\mathrm{O}$ espectro da desnutrição associada à infecção pelo HIV é amplo. A desnutrição pode ser detectada precocemente em crianças ou adultos infectados, porém ainda assintomáticos $(18,28)$. Estudos transversais e longitudinais demonstram que a prevalência da desnutrição é maior no grupo de crianças situadas na categoria clínica $\mathrm{C}$ da classificação do $\mathrm{CDC}(25)$, associada à maior gravidade da doença. $\mathrm{O}$ quadro do emaciamento é uma das principais manifestações de progressão da doença, sendo considerada na faixa etária pediátrica, em relato recente do CDC, o quarto indicador da Aids em termos de freqüência. O catabolismo é uma das principais manifestações da evolução da doença, classificado atualmente como o segundo indicador mais importante em crianças (29). Em razão da necessidade de sistematização da abordagem desse evento clínico, esse quadro de desnutrição especificamente associado ao HIV foi denominado wasting syndrome ou síndrome de emaciamento, sendo definido dessa forma conforme a idade (25). Os critérios diagnósticos estão apresentados na Tabela 1.

Para a denominação das formas mais graves de desnutrição, perda extrema de peso, apatia e redução da massa de tecido adiposo e muscular, o termo caquexia é utilizado com freqüência, de maneira análoga ao seu uso em outras doenças crônicas. A definição de caquexia associada ao HIV é bastante imprecisa, não havendo critério uniforme. A fisiopatologia do déficit de crescimento, do emaciamento e da caquexia não está bem determinada e, sem dúvida, depende de múltiplos fatores, embora o distúrbio endócrino seja um dos principais mecanismos (18).

\section{Síndrome lipodistrófica (SL) associada ao tratamento com anti-retrovirais}

A SL está associada, principalmente, a dois grupos de anti-retrovirais, os ITRNs e os IPs. Consiste de alterações na distribuição da gordura corporal, associada a 
Tabela 1. Critérios diagnósticos da síndrome de emaciamento em adultos e crianças $(18,25)$.

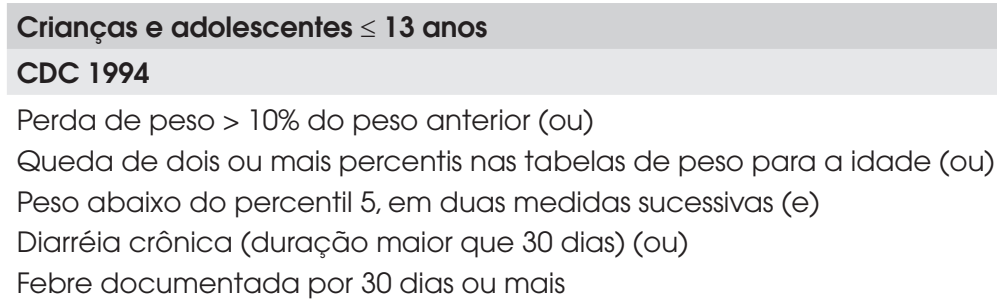

Perda de peso involuntária de mais de 10\% do peso basal (e) Diarréia crônica ( $\geq 30$ dias) (ou)

Astenia e febre documentada ( $\geq 30$ dias, constante ou intermitente), na ausência de doença ou condição que possa explicar tais sinais (câncer, tuberculose, criptosporidiose etc.)

\author{
Presença de qualquer um dos critérios adiante: \\ 10\% de perda de peso não-intencional em 1 ano (ou) \\ $7,5 \%$ de perda de peso não intencional em 6 meses \\ (ou) \\ 5\% de perda de peso não intencional em 3 meses \\ Presença de qualquer um dos dados auxiliares adiante: \\ Em homens: massa celular corpórea* < 35\% do peso \\ corporal total se IMC < 27 (ou) \\ Em mulheres: massa celular corpórea" $<23 \%$ do peso \\ corporal total se IMC $<27$
}

(ou) $\mathrm{IMC}<20$

$\overline{C D C}=$ Centers for Disease Control; IMC = índice de massa corporal; *medida por bioimpedância elétrica.

anormalidades bioquímicas (dislipidemia, alteração na homeostase da glicose, hiperlactatemia e desmineralização óssea). Alguns grupos de pesquisa não incluem a hiperlactatemia e a desmineralização óssea neste conjunto. Não há evidências conclusivas sobre a associação entre os agentes anti-retrovirais e a desmineralização óssea, sendo tal anormalidade comum entre pacientes pediátricos e adultos. A prevalência da hiperlactatemia varia conforme os modelos de estudo, estando associada à própria patogênese da Aids. É importante destacar que a infecção pelo HIV, em sua história natural, pode estar associada a todas as manifestações mencionadas anteriormente. Tal constatação destaca a importância da inflamação crônica, com desregulação da rede de citocinas, na patogênese da SL $(11,30)$.

Clinicamente, a alteração na distribuição da gordura corporal pode ser classificada em três categorias $(11,30)$ : a) lipoatrofia: redução da gordura em regiões periféricas, como braços, pernas e face; b) lipohipertrofia: acúmulo de gordura em região abdominal, presença de gibosidade dorsal; c) forma mista: caracterizada pela associação de componentes das duas formas anteriormente descritas.
A lipoatrofia é mais associada ao uso de ITRN, em especial a estavudina (D4T), enquanto a lipohipertrofia está associada tanto ao ITRN quanto ao IP. A alteração na forma corporal, resultante desse processo, é causa de grande desconforto aos pacientes, estando associada a depressão, baixa auto-estima, dificuldades na vida sexual e redução na adesão ao tratamento anti-retroviral, particularmente entre adolescentes e adultos jovens. A reversibilidade de tais alterações, com a suspensão ou mudança dos medicamentos, é variável, sendo menor nas formas com lipoatrofia $(11,30)$.

A dislipidemia, caracterizada pelo aumento do LDL-colesterol, diminuição do HDL-colesterol e/ou aumento de triglicérides, está particularmente associada aos IPs, mas a avaliação da prevalência está comprometida por diferenças nos modelos de estudo. Um estudo longitudinal com amostra significativa relata a presença de, ao menos, uma das anormalidades laboratoriais em $49 \%$ de pacientes de uma coorte adulta (31). Estudos transversais pediátricos revelam variação de $20 \%$ (um único IP) até 90\% (dois ou mais IPs) (27). As alterações no metabolismo de carboidratos (hiperinsulinemia e hiperglicemia) também são associadas aos IPs. 
Entretanto, não existem ainda dados sobre aumento de fatores de risco cardiovascular em crianças. O conjunto de alterações clínicas e laboratoriais identificado na SL mostra semelhanças com a síndrome metabólica, observada tanto em adultos quanto em crianças não infectadas, e está associada à morbidade significativa (32) (Tabela 2).

Tabela 2. Semelhanças entre a síndrome lipodistrófica (SL) do HIV/Aids e a síndrome metabólica (1 1).

\begin{tabular}{llc}
\hline Parâmetro & $\begin{array}{c}\text { Síndrome } \\
\text { metabólica }\end{array}$ & $\begin{array}{c}\text { Síndrome } \\
\text { lipodistrófica } \\
\text { (HIV/Aids) }\end{array}$ \\
\hline Hiperinsulinemia & Presente & Presente \\
\hline Obtolerância à glicose & Presente & Presente \\
\hline Hipertensão & Presente & Presente \\
\hline Dislipidemia* & Presente & Presente \\
\hline Hiperuricemia & Presente & Presente \\
\hline Estado pró-trombótico & Presente & Presente \\
\hline Estado pró-inflamatório & Presente & Presente \\
\hline
\end{tabular}

* LDL - colesterol e triglicérides elevados; HDL - colesterol diminuído.

\section{DISTÚRBIOS ENDÓCRINO-IMETABÓLICOS}

Vários estudos confirmam que todos os tecidos humanos podem ser afetados pelo HIV, incluindo o sistema endócrino (33-35). É importante observar que alguns dos sinais e sintomas da Aids, como náuseas, vômitos, anorexia, emagrecimento, hiperpigmentação, febre, diarréia, hipotensão ortostática, também são manifestações de insuficiência adrenal. O retardo de crescimento nas crianças infectadas também pode se assemelhar ao das doenças endócrinas (33).

Vários relatos sobre endocrinopatias nos pacientes com HIV e Aids têm sido publicados, mas a heterogeneidade dos dados não permite que se especifique um perfil de alterações hormonais que caracterize esses pacientes. Um aspecto a ser considerado é que muitas pesquisas não avaliam número significativo de pacientes, ou não têm homogeneidade em relação ao uso de medicações e ao grau de comprometimento clínico entre os pacientes estudados. Por outro lado, o próprio padrão episódico da secreção hormonal é também responsável por grande variação nos resultados, principalmente quando as amostras de sangue são coletadas aleatoriamente $(29,35,36)$ As alterações hormonais são, provavelmente, causadas pela própria infecção viral ou secundárias ao comprometimento das glândulas endócrinas, seja por infecções oportunistas ou por medicamentos para tratar as infecções ou suas complicações (4,33-35).

A infecção pelo HIV leva à perda progressiva da função imune, com diminuição no número de células $\mathrm{T}$, CD4, concomitante às alterações na função das células $\mathrm{T}$ e das principais vias regulatórias da imunidade. A expressão das citocinas, incluindo a interleucina-l (IL-1) e a interleucina-6 (IL-6), o fator de necrose tumoral- $\alpha$ (TNF- $\alpha$ ) e o interferon, está alterada. As citocinas influenciam o metabolismo intermediário de várias formas, incluindo o efeito sobre os adipócitos, que têm receptores para TNF- $\alpha$, sendo inclusive por eles produzido. Por essa razão, a infecção crônica leva às alterações metabólicas características, entre as quais o aumento na mobilização de gordura, com supressão da oxidação pela inibição da lipase lipoprotéica (4). Alguns estudos demonstram que, sob a influência de mecanismo mediado por citocinas, a primeira manifestação do comprometimento do sistema endócrino pode ser a alteração na regulação do relógio biológico, responsável pelo controle da secreção hormonal do eixo hipotalâmico-hipofisário, e que precede seus sinais e sintomas (36).

\section{Tireóide}

Amplo espectro de alterações tireoidianas pode ocorrer em pacientes soropositivos e com Aids, embora não estejam presentes de maneira consistente. São relatadas disfunções na secreção basal de hormônio tireoestimulante (TSH), hipotireoidismo subclínico e eutireoidismo do paciente enfermo. A avaliação da secreção circadiana de TSH demonstra diminuição significativa do pico noturno (à meia-noite), considerado índice sensível para avaliação de hipotireoidismo central (33). Essa alteração indica disfunção na regulação hipotalâmica-hipofisária e precede quadro mais expressivo de hipotireoidismo (33-36).

As alterações tireoidianas resultam do comprometimento sistêmico, da doença hepática, da deficiência nutricional e da alteração das citocinas. A IL-1, a IL-6, assim como o TNF- $\alpha$, inibem a atividade das deiodinases (tipo 15 '), o que resulta a diminuição da triiodotironina $\left(\mathrm{T}_{3}\right)$ plasmática, níveis variáveis de tetraiodotironina $\left(\mathrm{T}_{4}\right)$, com TSH normal ou com discreta elevação (35). Alterações na expressão do neuropeptídeo Y (NPY) no núcleo infundibular podem alterar as células produtoras 
de hormônio liberador de TSH (TRH) e causar a diminuição do TSH em alguns pacientes $(35,37)$. Para alguns autores, pode haver relação linear entre os níveis de $\mathrm{T}_{3}, \mathrm{~T}_{4}$ livre e a porcentagem de linfócitos $\mathrm{T}$, com correlação entre as anormalidades bioquímicas e a gravidade da doença, tanto em crianças quanto em adultos (4).

\section{Adrenal}

Em relação ao eixo adrenal, os níveis basais de hormônio adrenocorticotrófico (ACTH) e cortisol estão dentro da normalidade, mostrando que a organização fisiológica circadiana do cortisol está mantida nos pacientes soropositivos. Os principais estudos demonstram que a prevalência de alterações da adrenal não é muito elevada nos pacientes com HIV. No entanto, alteração adrenal na forma de insuficiência pode ser observada em pacientes com a síndrome do emaciamento durante tratamento com antifúngicos ou associada à deficiência de crescimento. Nesses casos, a principal manifestação é a produção anormal de cortisol e/ou a redução na produção de andrógenos, principalmente nos adultos infectados. Embora o antifúngico cetoconazol interfira com a esteroidogênese, parece que esse efeito não prejudica a produção de cortisol em doses padronizadas. Níveis elevados de cortisol também já foram descritos como resultado do efeito inibitório da desnutrição no metabolismo do cortisol $(35,38)$.

Existe ainda relação inversa entre a razão diidroepiandrosterona (DHEA)/cortisol e o z-escore do índice de massa corporal (IMC) e do peso, sugerindo que crianças com baixos índices têm maior porcentagem de gordura. Nos adultos infectados, esse índice se correlaciona com a massa magra, tanto em homens quanto em mulheres, enquanto nos homens essa correlação ocorre apenas com o IMC e a massa gorda. Existe ainda relação inversa entre a razão DHEA/cortisol e o z-escore da estatura em crianças infectadas ou não. O significado biológico dessa diferença ainda não está claro (29).

A relação entre os níveis de DHEA e o grau de imunossupressão é ainda controversa. Em crianças não são descritas correlações entre a porcentagem de linfócitos e os níveis de cortisol, enquanto em adultos com insuficiência adrenal essa relação parece ser positiva $(29,35,36)$.

\section{Crescimento}

Os primeiros estudos em crianças infectadas pelo HIV e/ou crianças com Aids demonstraram prevalência sig- nificativa de distúrbios do crescimento linear, que se apresentam de diferentes formas, desde a dificuldade para crescer (failure to thrive), passando pelo peso e pela estatura abaixo do padrão normal para a idade, até a condição de interrupção do crescimento e síndrome de emaciamento $(29,33)$. Estudos realizados na década de 1990, com crianças sintomáticas infectadas, demonstraram que o retardo de crescimento precedia tanto o declínio na porcentagem de linfócitos CD4 quanto o desenvolvimento de infecções oportunistas. Já que o comprometimento no crescimento linear e o baixo ganho de peso são concomitantes à progressão da doença, estes passaram a ser considerados fatores prognósticos para a sobrevida (39). Nas crianças imunossuprimidas, a relação entre carga viral, crescimento e sobrevida pode não ser tão evidente quanto nas crianças imunocompetentes ou assintomáticas (17).

A maioria das crianças infectadas durante a gestação, quando comparadas às crianças nascidas sem infecção (em mães soropositivas), apresenta retardo de crescimento intra-uterino ( $25 \%$ a $50 \%$ ), com comprometimento precoce do perímetro cefálico, do comprimento e do peso ao nascer (33). O comprometimento da estatura e do ganho de peso é geralmente precoce, sem crescimento de recuperação (catch up) ou crescimento estável no período pós-natal, mostrando perda progressiva em relação à curva normal de crescimento. As crianças com HIV infectadas no período pós-natal também apresentam crescimento linear comprometido, assim como baixo ganho de peso e retardo na maturação óssea $(17,29)$.

No contexto da Aids, assim como em outras doenças crônicas, a deficiência de crescimento depende de múltiplos fatores, entre os quais, desnutrição, distúrbios gastrintestinais (má-absorção), estresse, doença crônica e distúrbios endócrinos. O principal componente da desnutrição é o aporte nutricional inadequado, secundário à anorexia, lesões orais ou do trato digestivo alto (causa infecciosa), redução de ingestão (causa psicológica ou econômica) ou ainda má-absorção de nutrientes (diarréia crônica). Associam-se a esses fatores a aceleração do catabolismo protéico e o aumento do gasto metabólico, secundários à replicação viral não-controlada e à resposta inflamatória dela decorrente (incluindo desregulação da rede de citocinas), bem como à presença de infecções oportunistas ou neoplasias secundárias à imunossupressão. Alguns trabalhos, no entanto, discordam em relação à possível influência do estado hipermetabólico na deficiência de crescimento de crianças infectadas $(7,35)$. 
Ainda existem dúvidas no que diz respeito aos efeitos dos medicamentos anti-retrovirais no crescimento das crianças infectadas. Um estudo randomizado com 197 crianças clinicamente estáveis (de 2 a 17 anos), e submetidas à terapia com anti-retrovirais (ritonavir), comprovou estatura abaixo da média (z-escore médio de $-0,57$ desvios-padrão [DP]), sem apresentar correlação com o controle da replicação viral. As crianças que atingiram a melhor resposta viral foram as que tiveram o pior crescimento. A alteração nos níveis de fator de crescimento insulina-like 1 (IGF-1) pode ser resultante da alteração na produção de proteína ligadora de IGF do tipo l (IGFBP-1), que está diminuída nas crianças infectadas no início do tratamento com anti-retrovirais, ou pela diminuição da disponibilidade plasmática de IGF-1, causada pelo tratamento combinado (17). Permanece em discussão se intervenções nutricionais ou a terapia com anticitocinas, ou ainda com hrGH e IGF-l, poderiam ser opções para melhorar o crescimento e a progressão da doença nesses pacientes (40).

\section{Regulação do eixo hormônio de crescimento/fator de crescimento insulina-like 1 (GH/IGF-1)}

A relação entre GH, IGFs, infecção por HIV e Aids é muito complexa, à semelhança do que ocorre em pacientes com outras doenças graves. A Figura 1 resume alguns dos aspectos dessa interação. Embora alguns investigadores já tenham sugerido papel direto de IGF-1 no processo de infecção pelo HIV, modulando a produção de células $\mathrm{T}$ ou interferindo com a replicação de HIV, o estado nutricional e a composição corporal são os determinantes mais importantes da fisiologia do $\mathrm{GH}$, e a principal conexão entre IGFs e a infecção por HIV. No entanto, o mecanismo pelo qual essas alterações ocorrem não está bem determinado e as controvérsias ainda persistem (42).

Para alguns pesquisadores, ocorre um quadro de resistência ao GH, caracterizado por elevação na concentração de GH e diminuição nos níveis de IGF-1. A ausência de resposta após hrGH na dose de $0,1 \mathrm{mg} / \mathrm{kg} /$ dia, tra-

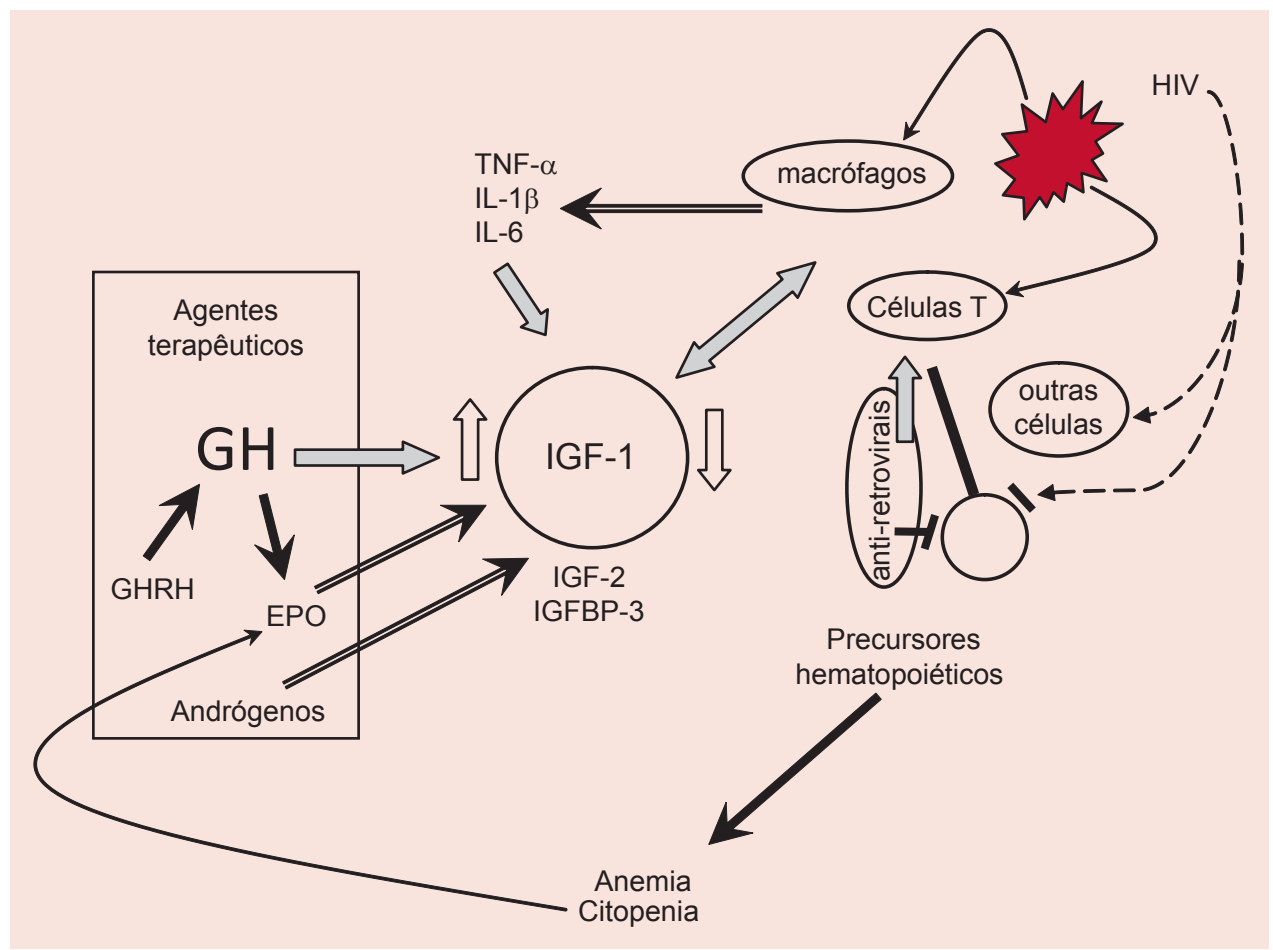

GH = hormônio de crescimento; GHRH = hormônio liberador de GH; EPO = eritropoitina; IGF-1 = fator insulina-like tipo 1; IGF-2 = fator insulina-like tipo 2; IGFBP-3 = proteína carreadora de IGF tipo 3: TNF- $\alpha=$ fator de necrose tumoral alfa; $\|$ = interleucina. Adaptado da referência 41.

Figura 1. Relações entre IGF-1 e a infecção pelo HIV. Representação esquemática dos principais fatores que influenciam as concentrações de IGFs após a infecção pelo HIV (que diminui os níveis de IGF-1), a interação entre IGF e o sistema hematopoiético e os agentes terapêuticos, os quais elevam as concentrações de IGF-1. 
duzida pela produção de IGF-1 em pacientes com Aids e emaciamento, é consistente com o conceito de resistência hormonal (8).

À semelhança do que já foi descrito em relação a outros hormônios, e conforme já foi discutido nesse texto, o estado nutricional compromete a produção de GH de diversas formas. A desnutrição afeta de maneira significativa a produção das proteínas carreadoras de IGF-1, que regulam o transporte, a liberação e a ação da fração livre de IGF-1. Foi demonstrado em pacientes infectados, com mais de $10 \%$ de decréscimo no peso corporal ideal, redução de $55 \%$ nos níveis de IGF-1, proteína ligadora de IGF do tipo 3 (IGFBP-3) e redução da habilidade de formar complexo ternário entre IGF-1, IGFBP-3 e a subunidade ácido lábil (ALS) $(8,43)$. Nessas condições, ocorre aumento da proteólise, elevação do nitrogênio urinário, diminuição da síntese protéica, com aumento do turnover, aumento da síntese hepática de proteínas (resposta inflamatória aguda) e diminuição nos níveis de aminoácidos de cadeia longa. O estado catabólico inflamatório retira aminoácidos essenciais do músculo esquelético por meio da gliconeogênese hepática. A redução na proteína carreadora do $\mathrm{GH}$ (GHBP), que é parte do domínio extracelular do receptor de $\mathrm{GH}$, corresponde à alteração do receptor, contribuindo para a resistência ao $\mathrm{GH}$, que também está relacionada a alterações pós-receptor. A proteólise aumentada de IGFBP-3 também tem sido descrita nos pacientes pediátricos com HIV e síndrome de emaciamento com retardo de crescimento. Nesses pacientes, as mudanças na composição corporal características da caquexia da Aids também contribuem para a resistência ao GH e para o retardo de crescimento, especialmente quando ocorre redução na massa magra. O estudo de crianças infectadas $(\mathrm{n}=2 \mathrm{l})$ no período gestacional, com baixo peso e comprometimento da estatura, comparadas a crianças expostas às mesmas condições gestacionais (drogas ilícitas), demonstrou correlação dos níveis hormonais de IGFBP-3, $\mathrm{T}_{3} \mathrm{e}$ razão DHEA/cortisol com o peso e o IMC, o que indica efeito somático mais do que efeito isolado no crescimento linear (29). Apenas nas crianças infectadas com baixo peso e IMC/idade, os níveis de IGFBP-3 estavam diminuídos de maneira significativa, enquanto a secreção de GH foi semelhante nos dois grupos. Os autores concluíram que, nas crianças infectadas, a resistência ao GH, assim como a modificação na síntese dos precursores dos andrógenos da adrenal na produção de cortisol, contribuem para a alteração na composição corporal e na interrupção do crescimento (29). A alteração na regula- ção do $\mathrm{GH}$ pode também decorrer da própria infecção pelo HIV, que compromete a produção de IGFBP-1, ou por co-infecções agudas ou crônicas, ou ainda outras anormalidades metabólicas (já discutidas na seção Crescimento). A contribuição específica das citocinas está sob avaliação, mas pesquisas realizadas em crianças infectadas questionam a possibilidade da IL-6 ser inversamente relacionada à produção de IGF-1, o que estabelece ligação entre a deficiência no crescimento e o aumento na produção de interleucinas, à semelhança do que ocorre na síndrome catabólica dos adultos, relacionada ao aumento na produção de TNF- $\alpha$ (29).

Existem dúvidas se a infecção per si poderia influenciar o funcionamento do eixo GH/IGF-1, mas de acordo com estudo que comparou homens adultos infectados, assintomáticos e indivíduos com Aids, com peso estável e sem infecções oportunistas, a controles saudáveis, não se demonstrou diferenças no padrão de secreção de GH durante 24 horas (pico, amplitude em freqüência e intervalo) entre os grupos, assim como nos níveis de IGF-1 ou IGFBP-3 (44). Para outros autores, a proteína que se dissocia do HIV-1, e é homóloga ao hormônio liberador do GH (GHRH), se liga aos receptores hipofisários, potencialmente suprimindo a liberação de $\mathrm{GH}$ (8). A avaliação dos níveis de fator de crescimento insulina-like tipo 2 (IGF-2) mostra um índice que apresenta correlação significativa com o grau de progressão da doença nos pacientes infectados, em caquexia. Os pacientes adultos com Aids e síndrome de emaciamento são os que apresentam os menores valores de IGF-2. A avaliação dos níveis de IGF-1 nos pacientes sem caquexia demonstra não haver diferenças reais no padrão de secreção de $\mathrm{GH}$, tanto nos pacientes infectados assintomáticos quanto nos pacientes com Aids sem catabolismo, em comparação aos controles (41). Alguns estudos em crianças estão em desacordo com os achados em adultos e consideram a secreção de GH normal, contrariando a hipótese de resistência hormonal para justificar a diminuição nos níveis de IGF-1. Portanto, parece que ocorre ausência de sincronização na liberação do GH e IGF, indicando desarranjo hipotalâmico-hipofisário (45).

A avaliação da relação entre GH/IGF-1 e lipodistrofia apresenta resultados controversos. Alguns estudos confirmam a diminuição na secreção de IGF-1, com diminuição na amplitude dos pulsos de secreção do GH (freqüência normal) nos pacientes que apresentam lipodistrofia. Atribuiu-se essa alteração às anormalidades na composição corporal e às anormalidades 
metabólicas, especialmente o aumento na gordura visceral e nos ácidos graxos livres. $\mathrm{O}$ aumento na somatostatina, com diminuição da ghrelina, também contribui para a diminuição do $\mathrm{GH}$ (46). Contudo, estudo realizado com número significativo de pacientes demonstra que a lipodistrofia não interfere com os níveis de IGF-1, que foram comparáveis aos dos controles normais (47). Embora os objetivos e os resultados dos diversos trabalhos seja bem heterogêneo, fica bastante evidente, nos estudos realizados em crianças e adolescentes, que os níveis de IGF-1 estão diminuídos nas crianças com Aids, promovendo alteração do crescimento, em comparação aos controles (48).

Todas as alterações descritas em relação ao $\mathrm{GH} /$ IGF-1, consideradas em conjunto, sugerem que ocorrem inúmeras adaptações na secreção de $\mathrm{GH}$, na dependência do estado nutricional e na composição corporal nos pacientes com HIV que desenvolvem Aids. Nos pacientes infectados, mas saudáveis, essas alterações não são descritas. A infecção por HIV está geralmente associada à diminuição nos níveis de IGF-l, em particular nas crianças com caquexia. Na lipodistrofia, os valores de IGF-1 são geralmente normais, ou discretamente diminuídos, em comparação às crianças não infectadas (4l).

\section{O USO DO HORMÔNIO DE CRESCIMENTO}

\section{Tratamento da síndrome de emaciamento}

As inúmeras pesquisas que investigam as modificações na regulação do sistema $\mathrm{GH} / \mathrm{IGF}-1$, após a infecção pelo HIV e o desenvolvimento da Aids, permitiram a elaboração de intervenções terapêuticas, usando hrGH ou IGF-1, e até o GHRH, no tratamento de algumas condições associadas à doença, como perda de peso e lipodistrofia (41).

As pesquisas usando o hrGH em curto prazo em pacientes com diversos estados catabólicos, como queimados, pós-operatório, síndrome do intestino curto, doença celíaca e câncer, resultaram aumento da massa magra, melhora no balanço do nitrogênio, na síntese protéica e na oxidação lipídica. $\mathrm{O}$ emprego do hrGH foi inicialmente proposto para o tratamento da síndrome de emaciamento, a fim de aumentar a massa magra e a massa muscular, sendo essa indicação aprovada pela Food and Drug Administration (FDA) (49).

Os efeitos anabólicos e anticatabólicos do hrGH foram avaliados em diferentes estudos farmacodinâmi- cos, realizados em pacientes com HIV e síndrome de emaciamento. Um dos estudos avaliou a melhora na síntese protéica muscular em relação à gravidade da doença, após hrGH na dose de $6 \mathrm{mg} /$ dia durante duas semanas (8). Outra pesquisa demonstrou em seis pacientes HIV positivos com síndrome da caquexia aumento significativo do peso corporal, da oxidação de lipídeos e carboidratos e das concentrações de IGF-1, com diminuição na excreção urinária de nitrogênio e oxidação de proteínas, após a administração do hrGH durante sete dias, na dose de $0,1 \mathrm{mg} / \mathrm{kg} /$ dia. O máximo da melhora ocorreu no sétimo dia de tratamento $(5,8)$. O uso do hrGH na dose de $5 \mathrm{mg} /$ dia, durante 12 semanas, promoveu melhora na massa magra, no peso e redução na gordura corporal dos pacientes em caquexia. No entanto, o mesmo efeito não foi obtido quando doses inferiores de hrGH foram utilizadas $(2,5$ $\mathrm{mg} /$ dia). Por outro lado, seis semanas após a interrupção da medicação, os efeitos benéficos do tratamento desapareceram $(8,50)$. Foi possível comprovar que o hrGH na dose de $6 \mathrm{mg} /$ dia, durante duas semanas, aumenta a síntese protéica, o peso corporal e os níveis de IGF-1, mas a intensidade da resposta à terapêutica é dependente da gravidade da doença (51). O aumento da massa muscular foi menor nos pacientes com a forma mais grave da doença. Os pacientes com Aids e mais de $10 \%$ de perda de peso tiveram $42 \%$ de decréscimo na taxa de síntese protéica, embora tenham elevado os níveis de IGF em resposta ao hrGH. Os autores concluíram que não é possível determinar se a inabilidade do músculo em responder ao hrGH no grupo com Aids (e mais de $10 \%$ de perda de peso) representa insensibilidade ao hrGH no músculo ou falha para responder à elevação de IGF-1, ou seja, resistência a IGF (8). O aumento da massa magra e a diminuição da gordura são os efeitos do hrGH relacionados às modificações na composição corporal nos pacientes com a síndrome do emaciamento. A maior perda de gordura ocorre especificamente na região do tronco, sendo mínima em outras áreas, o que afasta a preocupação com possível exacerbação da lipoatrofia, característica dos pacientes submetidos à terapia com anti-retrovirais, caso sejam tratados com hrGH. Os principais efeitos metabólicos do tratamento compreendem aumento do metabolismo basal, do catabolismo lipídico, de IGF-1 e da glicose plasmática $(5,8)$.

Diversos estudos controlados, randomizados, duplocego e com duração de 12 semanas foram realizados tanto antes quanto durante a terapêutica combinada 
(HAART), a fim de determinar o efeito do hrGH na síndrome do emaciamento. A Tabela 3 resume as principais características desses estudos. De modo geral, considerou-se $10 \%$ de perda de peso como critério para o emaciamento, mas o número de pacientes, além da dose de hrGH foi bastante variável. Os resultados dos estudos realizados foram benéficos na sua maioria, no que diz respeito a ganho de peso e a aumento de massa magra, além de melhora na qualidade de vida em alguns. Um dos principais estudos clínicos, realizado no período anterior ao tratamento com anti-retrovirais, avaliou a resposta de 178 pacientes infectados pelo HIV ao hrGH, durante 12 semanas, e foi utilizado para a aprovação do uso do hrGH em pacientes com emacia- mento (49). A dose de hrGH utilizada foi de $0,1 \mathrm{mg} /$ $\mathrm{kg}$ peso/dia, média de $6 \mathrm{mg} /$ dia. Não foram observadas mudanças na contagem de linfócitos, evolução da doença ou da carga viral, sendo a medicação bem tolerada. A prevalência de efeitos adversos foi maior no grupo tratado, e 15 pacientes tiveram a dose de hrGH reduzida. A síndrome do túnel do carpo se manifestou em três pacientes e outros três interromperam o estudo por edema e artralgias $(n=2)$, enquanto um por aparecimento de sarcoma de Kaposi. Nos oito pacientes (quatro em cada grupo) com doença manifesta, não houve progressão do quadro. A elevação nos níveis de glicose, hemoglobina glicada e fósforo foi discretamente maior no grupo tratado em comparação ao controle (49).

Tabela 3. Estudos randomizados, duplo-cego, controlados, de 12 semanas com hormônio do crescimento recombinante humano $(\mathrm{hrGH})$ em pacientes com síndrome do emaciamento a associada ao HIV/Aids (adaptado da referência 8).

\begin{tabular}{|c|c|c|c|c|c|}
\hline Autores & Dose hrGH & $\begin{array}{l}\text { Número } \\
\text { pacientes } \\
\text { tratados }\end{array}$ & $\begin{array}{l}\text { Incremento de peso } \\
\text { em relação ao início } \\
\text { (kg) }\end{array}$ & $\begin{array}{l}\text { Incremento na massa } \\
\text { magra }^{\mathrm{b}}(\mathrm{kg})\end{array}$ & Outros achados \\
\hline \multicolumn{6}{|l|}{ Pré-HAART } \\
\hline $\begin{array}{l}\text { Waters e cols. } \\
(52)^{c}\end{array}$ & 1,4 mg/dia & 15 & $\begin{array}{l}\text { Significativo na } \\
\text { semana } 6(p<0,01) \\
\text { e NS na semana } 12\end{array}$ & $\begin{array}{l}\text { Significativo na semana } \\
6(p<0,01) \text { e NS na } \\
\text { semana } 12\end{array}$ & $\begin{array}{l}\text { Melhora da qualidade de } \\
\text { vida e força na semana } 12\end{array}$ \\
\hline $\begin{array}{l}\text { Schambelan e } \\
\text { cols. (49) }\end{array}$ & $0,1 \mathrm{mg} / \mathrm{kg} / \mathrm{dia}$ & 90 & $\begin{array}{l}+1,6 \text { na semana } 12 \\
(p<0,05 \text { versus } \\
\text { placebo })\end{array}$ & $\begin{array}{l}+3,0 \text { na semana } 12 \\
(p<0,001 \text { versus } \\
\text { placebo) }\end{array}$ & $\begin{array}{l}\text { Aumento na capacidade } \\
\text { para exercício na semana } 12 \\
\text { ( } p<0,05 \text { versus placebo), } \\
\text { sem diferença na qualidade } \\
\text { de vida }\end{array}$ \\
\hline \multicolumn{6}{|l|}{ Pós-HAART } \\
\hline $\begin{array}{l}\text { Esposito e cols. } \\
\text { (53) }\end{array}$ & $6 \mathrm{mg} / \mathrm{dia}$ & $12^{\mathrm{d}}$ & $\begin{array}{l}+1,5 \text { na semana } 12 \\
(p<0,01 \text { versus } \\
\text { placebo; } p<0,05 \\
\text { versus início) }\end{array}$ & $\begin{array}{l}+6,1 \% \text { na semana } 12 \\
(p<0,01 \text { versus } \\
\text { placebo; } p<0,05 \\
\text { versus início) }\end{array}$ & $\begin{array}{l}\text { Aumento na utilização/ } \\
\text { extração de oxigênio pelo } \\
\text { músculo na semana } 12(\mathrm{p}< \\
0,05 \text { versus início e placebo) }\end{array}$ \\
\hline $\begin{array}{l}\text { Moyle e cols. } \\
(54)\end{array}$ & $0,1 \mathrm{mg} / \mathrm{kg} / \mathrm{dia}$ & 166 & $\begin{array}{l}+2,96 \text { na semana } 12 \\
(p<0,001 \text { versus } \\
\text { placebo) }\end{array}$ & $\begin{array}{l}+5,21^{e} \text { na semana } 12 \\
(p<0,001 \text { versus } \\
\text { placebo) }\end{array}$ & $\begin{array}{l}\text { Aumento na capacidade de } \\
\text { exercício e na qualidade de } \\
\text { vida na semana } 12(p<0,05 \\
\text { versus placebo) }\end{array}$ \\
\hline $\begin{array}{l}\text { Storer e cols. } \\
\text { (55) }\end{array}$ & $6 \mathrm{mg} / \mathrm{dia}$ & 21 & Não reportado & $\begin{array}{l}+2,5 \text { na semana } 12 \\
(\mathrm{p}<0,05 \text { versus } \\
\text { placebo; NS versus } \\
\text { nandrolona) }\end{array}$ & $\begin{array}{l}\text { Força muscular com aumento } \\
\text { semelhante entre os três } \\
\text { grupos (hrGH versus placebo } \\
\text { versus nandrolona) na semana } \\
\text { 12; nandrolona aumentou a } \\
\text { qualidade de vida }(p<0,05 \\
\text { versus hrGH); hrGH diminuiu a } \\
\text { adiposidade visceral }(p<0,01 \\
\text { versus placebo e nandrolona) } \\
\text { e massa gorda ( } p<0,01 \text { versus } \\
\text { placebo) }\end{array}$ \\
\hline
\end{tabular}


O principal estudo clínico com hrGH realizado até o momento com pacientes com a síndrome do emaciamento $(\mathrm{n}=757$; controles $=199)$ incluiu $88 \%$ de pacientes em uso de HAART e, além dos resultados esperados em relação à composição corporal, também demonstra mudança muito significativa da capacidade física dos pacientes tratados $(8,54)$. À semelhança de outros estudos $(8,49,52-55)$, os efeitos colaterais incluem artralgias $(36,4 \%)$, mialgia $(30,4 \%)$, edema periférico $(26,1 \%)$, cefaléia $(12,6 \%)$, artrose $(10,7 \%)$, náusea $(9,1 \%)$ e parestesia $(7,9 \%)$. Além dos efeitos adversos já relatados, entre os pacientes com HAART e emaciamento, houve aparecimento de um caso de diabetes melito tipo 2 e hiperglicemia em pacientes previamente diabéticos $(\mathrm{n}=2)(54)$.

Os efeitos adversos diminuem com a redução da dose ou interrupção do hrGH. Doses em dias alternados também podem ser consideradas nos pacientes com reações e nos que apresentam risco aumentado para intolerância à glicose, mas a eficácia ainda é discutida. $\mathrm{O}$ estudo de Moyle e cols. (54) ampliou o período de tratamento em 12 semanas, mas o limite de 48 semanas ainda não foi avaliado. Não existem evidências de que o hrGH estimule o aparecimento de tumores, embora nos pacientes com Aids o uso prolongado não tenha sido avaliado. Nos pacientes tratados, os níveis de IGF-1 e IGFBP-3 geralmente se elevam acima ou no limite da normalidade. Estudos epidemiológicos avaliam que níveis elevados de IGFBP-3 podem proteger os pacientes do risco atribuído à elevação de IGF-l, que estimula o crescimento celular e inibe a apoptose (54). A monitorização é muito importante, especialmente nos pacientes com risco aumentado de tumor, particularmente na terapia prolongada.

A associação de IGF-l ao hrGH, para tratamento do emaciamento, não tem demonstrado efeitos anabólicos significativos, embora tenha sido demonstrado ganho de peso discreto em um dos estudos, esse não se manteve durante as 12 semanas de tratamento. Essa forma de tratamento ainda não está aprovada (8). Além disso, revisão sistemática da literatura, realizada apenas com estudos clínicos randomizados, em países desenvolvidos, demonstrou que outra estratégia para prevenir a perda de peso tem sido utilizar o hrGH nas infecções secundárias (8).

\section{Tratamento da síndrome lipodistrófica (SL)}

O efeito do hrGH na composição corporal justificou o uso em pacientes com lipodistrofia. Vários estudos clí- nicos foram realizados e comprovaram o aumento na massa magra, a diminuição na gordura abdominal e a melhora no perfil lipídico (elevação do HDL), mas diminuição na sensibilidade à insulina, já comprometida nesses pacientes (46). Entre os efeitos do hrGH, os estudos iniciais em adultos demonstraram que poderia reduzir a adiposidade da região dorso-cervical, freqüentemente encontrada em pacientes com lipodistrofia, e influenciar na melhora da qualidade de vida. Estudos realizados posteriormente avaliaram o efeito de $6 \mathrm{mg} /$ dia de hrGH em 20 pacientes e demonstraram redução de $50 \%$ na gordura abdominal, no entanto, com aumento significativo de $27 \%$ na glicemia de jejum (46). Utilizando dose semelhante, Wanke e cols. demonstraram redução na circunferência do quadril e cintura dos pacientes, mas efeitos adversos também foram relatados, entre os quais, mialgias, edema das mãos e hiperglicemia (56).

A dose ideal para o tratamento não está estabelecida. A dose de $6 \mathrm{mg} /$ dia, usada em pacientes com síndrome de emaciamento, é bastante elevada, mas foi escolhida tendo como parâmetro a necessidade de ultrapassar a resistência ao hrGH, característica da síndrome, mas que não está presente na lipodistrofia. Nessas condições, a sensibilidade ao GH parece ser normal ou até mesmo aumentada. Essa dose reduz a gordura visceral de maneira significativa, mas além da redução da sensibilidade à insulina, outros efeitos colaterais se manifestam, como artralgia, mialgias, hipoestesias e retenção hídrica, provavelmente relacionados à elevação de IGF-1 em níveis suprafisiológicos. A utilização do hrGH na dose de $4 \mathrm{mg} /$ dia diminui a gordura visceral ( $20 \%$ ou mais), mas também causa efeitos colaterais, além de diminuir a tolerância à glicose. A utilização em dias alternados parece reduzir, de maneira satisfatória, os efeitos adversos, sem comprometer a perda de gordura (5).

$\mathrm{Na}$ tentativa de melhorar a secreção de $\mathrm{GH}$ destes pacientes, tem sido tentada a associação do hrGH ao GHRH, que parece ser efetiva no sentido de reduzir a gordura visceral, mas também não mantém os benefícios com a suspensão da medicação. $\mathrm{O}$ GHRH não influencia a secreção de insulina, e normaliza a secreção endógena, aumentando a área de pulso de GH. Serão necessários outros estudos prolongados com ambos os tratamentos para a reavaliação dos efeitos e das doses a serem utilizadas (46).

Todos os benefícios obtidos durante o tratamento não persistem 12 semanas após a interrupção do hrGH, o que pode ser considerado fator limitante. Embora 
não existam dúvidas quanto aos efeitos positivos do hrGH nessas condições da lipodistrofia, a diminuição na sensibilidade à insulina limita bastante a eficácia. $\mathrm{O}$ uso do hrGH em pacientes com lipodistrofia ainda não está aprovado pelo FDA.

\section{Crianças e adolescentes}

Existem pelo menos dois aspectos terapêuticos benéficos para crianças infectadas com HIV relacionados ao uso do hrGH: o efeito anabólico, que contribui para a recuperação imunológica, e a melhora do crescimento linear. Não existem ainda definições padronizadas no que diz respeito à dose, à duração do tratamento e à seleção dos pacientes. Os efeitos anabólicos do hrGH em crianças, assim como o incremento do peso e estatura, estão bem estabelecidos, não apenas em relação aos pacientes com Aids, mas também em relação a outras doenças crônicas $(4,57)$. Hardin e cols., com o objetivo de avaliar o efeito do hrGH no crescimento linear e sua capacidade de reverter o catabolismo protéico, trataram seis crianças impúberes infectadas pelo HIV, com estatura e peso abaixo do percentil 10 , ingestão calórica adequada, com a dose de $0,3 \mathrm{mg} / \mathrm{kg} /$ semana, durante seis meses. Esse estudo demonstrou aumento significativo na massa magra, assim como redução no catabolismo protéico, além da aceleração da velocidade de crescimento de $3,9 \mathrm{~cm} /$ ano no início da terapia para $7,9 \mathrm{~cm}$ após o tratamento. A estatura pode ter sido modificada pelo uso do hrGH, mas também é importante considerar a possibilidade de que a melhora no catabolismo protéico talvez seja o principal fator de estímulo para o crescimento. Não foram relatados efeitos adversos do tratamento ou modificação na carga viral (7).

A introdução da HAART resultou melhora significativa na morbi e mortalidade das crianças infectadas, assim como recuperação do crescimento, atribuída inicialmente ao decréscimo da carga viral e ao efeito sobre outros fatores, como infecção crônica ou recorrente, ingestão calórica inadequada, má-absorção, gasto energético, diminuição na produção de citocinas, alteração no metabolismo das gorduras e doenças endócrinas (disfunção tireoidiana e deficiência de GH), que, sem dúvida, também desempenham importante papel na recuperação do crescimento. $\mathrm{O}$ mecanismo exato dessa recuperação não está totalmente elucidado e, mesmo em relação à recuperação estatural, ainda existem algumas questões não respondidas, pois várias crianças mantêm a estatura e o peso abaixo da média, mesmo com o controle da carga viral (40). Um estudo realizado com o objetivo de analisar a recuperação do crescimento nos pacientes em uso de HAART acompanhou 27 crianças infectadas, com média de idade de 5,5 anos, e demonstrou a restauração da sensibilidade ao $\mathrm{GH}$, com elevação nos níveis de IGF-1 e IGFBP-3 nos primeiros meses de tratamento, retornando ao estado anabólico. A elevação de IGF- I foi positivamente correlacionada ao ganho de massa corporal e estatura, diminuição da carga viral, manutenção da função tireoidiana e adrenal. No entanto, o efeito anabólico do tratamento com hrGH parece ser maior. Os autores têm como hipótese que a reversão no catabolismo protéico é a chave para melhorar o crescimento linear, e que algumas crianças que não respondem aos IPs terão benefícios com o hrGH (48).

Em conclusão, o hrGH, quando utilizado em adolescentes infectados com aumento da gordura abdominal, na dose de $0,028 \mathrm{mg} / \mathrm{kg} /$ dia, durante 24 semanas, promoveu incremento na estatura $(2,4 \mathrm{~cm})$ e decréscimo na massa gorda, especialmente intra-abdominal e no tronco e membros. Houve aumento nos níveis de IGF-1, mas os níveis glicêmicos, de triglicérides e do colesterol não apresentaram modificações significativas (9).

\section{Novas perspectivas}

Um estudo, muito recente, comprova o efeito do hrGH no sistema imunológico de pacientes infectados pelo HIV (em terapia com HAART), por meio do aumento da massa tímica (reverte a involução tímica) e pela produção de células $\mathrm{T}$ e a facilitação da recuperação periférica de células CD4 (30\% de aumento). Esses efeitos foram mantidos durante três meses após a retirada da medicação. Esses resultados, embora promissores, não podem ser extrapolados para pacientes não medicados, sendo necessários novos estudos para avaliar qual a correlação entre o aumento do timo e a proteção imunológica e para comprovar os benefícios clínicos (58).

\section{CONCLUSÕES}

O eixo GH/IGF-1 é um dos principais reguladores do metabolismo protéico muscular, que está alterado nos pacientes com a síndrome de emaciamento, caracterizada, particularmente nos adultos, pela "resistência" ao GH, que não estimula de maneira eficiente a produção de IGF-1.

A utilização de hrGH em pacientes com Aids está bem definida na condição em que é necessário estimular 
o anabolismo. Está bem estabelecido que o hrGH na dose média de $6 \mathrm{mg} /$ dia $(0,1 \mathrm{mg} / \mathrm{kg} /$ dia $)$, durante 12 semanas, aumenta o peso corporal e a massa magra nos pacientes com perda de peso superior a $10 \%$. Contudo, pode ocorrer alteração na tolerância à glicose nos pacientes em uso de terapia anti-retroviral. Os níveis de glicose devem, portanto, ser monitorados durante o tratamento. Outros efeitos colaterais que também podem aparecer são reversíveis com ajustes de dose ou suspensão da medicação. Nas crianças, pode ser feito o mesmo tipo de indicação, incluindo os pacientes que também apresentam comprometimento da estatura. No entanto, em relação a essa indicação faltam estudos clínicos bem elaborados para estabelecer como e quem pode efetivamente se beneficiar do tratamento. No que diz respeito à lipodistrofia, embora efeitos benéficos do hrGH tenham sido comprovados, a indicação ainda não está devidamente autorizada, e algumas controvérsias persistem.

Existem ainda várias questões relacionadas à terapia dos pacientes com Aids e hrGH, entre essas, o tempo ideal de tratamento, o custo-benefício, os riscos envolvidos no uso prolongado, ou freqüente, e o impacto ao longo prazo na sobrevivência. É necessária que a seleção dos pacientes com indicação para tratamento seja sempre individualizada e avaliadas as condições metabólicas, as necessidades clínicas e pessoais.

\section{REFERÊNCIAS}

1. Unaids. Aids Epidemic Update; 2007 December. Disponível em: http://data.unaids.org/pub/EPISlides/2007/2007_epiupdate_en.pdf.

2. Ministério da Saúde. Secretaria de Vigilância em Saúde. Programa Nacional de DST e Aids. Boletim Epidemiológico AidsDST. Brasília: Ministério da Saúde. 2007. ano IV, no 1.

3. Leonard EG, McComsey GA. Metabolic complications of antiretroviral therapy in children. Pediatr Infect Dis J. 2003;22:77-84.

4. Hirschfeld S. Use of human recombinant growth hormone and human recombinant insulin-like growth factor-l in patients with human immunodeficiency virus infection. Horm Res. 1996;46:215-21.

5. Mulligan K, Grunfeld C, Hellerstein MK, Neese RA, SchambeIan M. Anabolic effects of recombinant human growth hormone in patients with wasting associated with human immunodeficiency virus infection. J Clin Endocrinol Metab. 1993;77:956-62.

6. Mynarcik DC, Frost RA, Lang $\mathrm{CH}$, DeCristofaro $\mathrm{K}$, McNurlan MA, Garlick PJ, et al. Insulin-like growth factor system in patients with HIV infection: effect of exogenous growth hormone administration. J Acquir Immune Defic Syndr. 1999;22:49-55.

7. Hardin DS, Rice J, Doyle ME, Pavia A. Growth hormone improves protein catabolism and growth in prepubertal children with HIV infection. Clin Endocrinol (Oxf). 2005;63:259-62.
8. Gelato M, McNurlan M, Freedland E. Role of recombinant human growth hormone in HIV-associated wasting and cachexia: pathophysiology and rationale for treatment. Clin Ther. 2007;29:2269-88.

9. Viganò A, Mora S, Manzoni P, Schneider L, Beretta S, Molinaro $M$, et al. Effects of recombinant growth hormone on visceral fat accumulation: pilot study in human immunodeficiency virus-infected adolescents. J Clin Endocrinol Metab. 2005;90:4075-80.

10. Kotler DP, Muurahainen N, Grunfeld C, Wanke C, Thompson $M$, Saag $M$, et al. Effects of growth hormone on visceral adipose tissue and dyslipidemia in HIV, an erratum. J Acquir Immune Defic Syndr. 2006;43:378-80.

11. Falutz J. Therapy insight: body-shape changes and metabolic complications associated with HIV and highly active antiretroviral therapy. Nat Clin Pract Endocrinol Metab. 2007;3:651-61.

12. Tesselaar K, Miedema F. Growth hormone resurrects adult human thymus during HIV-1 infection. J Clin Invest. 2008;3:844-7.

13. Lo JC, Mulligan K, Noor MA, Schwarz JM, Halvorsen RA Grunfeld C, et al. The effects of recombinant human growth hormone on body composition and glucose metabolism in HIV-infected patients with fat accumulation. J Clin Endocrinol Metab. 2001;86:3480-7.

14. European Paediatric Lipodystrophy Group. Antiretroviral therapy, fat redistribution and hyperlipidaemia in HIV-infected children in Europe. Aids. 2004;18:1443-51.

15. Ministério da Saúde. Brasil 2004. Secretaria de Vigilância em Saúde. Programa Nacional de DST e Aids Brasília (PN DSTAids). Série Manuais no 60. [atualização não disponível; acesso em 2008 Apr 28]. Disponível em: http://www.aids.gov.br.

16. HIV Trialists' Collaborative Group. Zidovudine, didanosine, and zalcitabine in the treatment of HIV infection: meta-analyses of the randomised evidence. Lancet. 1999;353:2014-25.

17. Chantry C, Byrd R, Englund J, Baker CJ, McKinney RE Jr. Pediatric Aids Clinical Trials Group Protocol 152 Study Team. Growth, survival and viral load in childhood HIV infection. Pediatr Infect Dis J. 2003;22:1033-9.

18. Wanke C, Kotler D. The HIV wasting collaborative consensus committee. Collaborative recommendations. The approach to diagnosis and treatment of HIV wasting. J Acquir Immune Defic Syndr. 2004;37 Suppl 5: S284-8.

19. Burgoyne RW, Tan DH. Prolongation and quality of life for HIV-infected adults treated with highly active antiretroviral therapy (HAART): a balancing act. J Antimicrob Chemother. 2008;61:469-73.

20. Dreimane D, Nielsen K, Deveikis A, Bryson YJ, Geffner ME. Effect of protease inhibitors combined with standard antiretroviral therapy on linear growth and weight gain in human immunodeficiency virus type 1-infected children. Pediatr Infect Dis J. 2001;20:315-6.

21. Verweel G, Van Rossum AMC, Hartwig NG, Wolfs T, Scherpbier $\mathrm{HJ}$, de Groot $\mathrm{R}$. Treatment with highly active antiretroviral therapy in human immunodeficiency virus type-1-infected children is associated with a sustained effect on growth. Pediatrics. 2002;109:E25.

22. Sterne JA, Hernán MA, Ledergerber B, Tilling K, Weber R, Sendi $P$, et al. Swiss HIV cohort study. Long-term effectiveness of potent antiretroviral therapy in preventing Aids and death: a prospective cohort study. Lancet. 2005;366:378-84.

23. Patel K, Hernán MA, Williams PL, Seeger JD, McIntosh K, Van Dyke RB, et al. Pediatric Aids Clinical Trials Group 219/219C Study Team. Long-term effectiveness of highly active antiretroviral therapy on the survival of children and adolescents 
with HIV infection: a 10-year follow-up study. Clin Infect Dis. 2008;46:507-15.

24. Scevola D, DiMatteo A, Giglio O, Uberti F. HIV infection-related cachexia and lipodystrophy. In: Mantovani G, editor. Cachexia and wasting: a modern approach. Milan: Springer; 2006. p. 407-28.

25. Centers for Disease Control and Prevention. 1994 revised classification system for human immunodeficiency virus infection in children less than 13 years of age. MMWR Morb Mortal Wkly Rep. 1994;43:1-10.

26. Mangili A, Murman DH, Zampini AM, Wanke CA. Nutrition and HIV infection: review of weight loss and wasting in the era of highly active antiretroviral therapy from the nutrition for healthy living cohort. Clin Infect Dis. 2006;42:836-42.

27. Leonard EG, McComsey GA. Antiretroviral therapy in HIV-infected children: the metabolic cost of improved survival. Infect Dis Clin North Am. 2005;19:713-29.

28. Polo R, Gómez-Candela C, Miralles C, Locutura J, Alvarez J, Barreiro $F$, et al. SPNS/GEAM/SENBA/SENPE/AEDN/SEDCA/ GESIDA. Recommendations from SPNS/GEAM/SENBA/ SENPE/AEDN/SEDCA/GESIDA on nutrition in the HIV-infected patient. Nutr Hosp. 2007;22:229-43.

29. Chantry CJ, Frederick MM, Meyer WA 3rd, Handelsman E, Rich K, Paul ME, et al. Endocrine abnormalities and impaired growth in human immunodeficiency virus-infected children. Pediatr Infect Dis J. 2007;26:53-60.

30. Valente AMM, Reis AF, Machado DM, Succi RCM, Chacra AR. Alterações metabólicas da síndrome lipodistrófica do HIV. Arq Bras Endocrinol Metab. 2005;49:871-81.

31. Saint-Marc T, Partisani M, Poizot-Martin I, Bruno F, Rouviere O, Lang JM, et al. A syndrome of peripheral fat wasting (lipodystrophy) in patients receiving long-term nucleoside analogue therapy. AIDS. 1999;13:1659-67.

32. Lundgren JD, Battegay M, Behrens G, De Wit S, Guaraldi G, Katlama C, et al. EACS Executive Committee. European Aids Clinical Society (EACS) guidelines on the prevention and management of metabolic diseases in HIV. HIV Medicine. 2008;9:72-81.

33. Laue L, Pizzo PA, Butler K, Cutler GB Jr. Growth and neuroendocrine dysfunction in children with acquired immunodeficiency syndrome. J Pediatr. 1990;117:541-5.

34. Matarazzo P, Palomba E, Lala R, Ciuti E, Altare F, de Sanctis L, et al. Growth impairment, IGF I hyposecretion and thyroid dysfunction in children with perinatal HIV-1 infection. Acta Paediatr. 1994;83:1029-34.

35. Panamonta O, Kosalaraksa P, Thinkhamrop B, Kirdpon W, Ingchanin $C$, Lumbiganon P. Endocrine function in Thai children infected with human immunodeficiency virus. J Pediatr Endocrinol Metab. 2004;17:33-40.

36. Rondanelli M, Caselli D, Aricò M, Maccabruni A, Magnani B, Bacchella L, et al. Insulin-like growth factor I (IGF-I) and IGF-binding protein 3 response to growth hormone is impaired in HIV-infected children. Aids Res Hum Retroviruses. 2002;18:331-9.

37. Fliers $E$, Unmehopa UA, Manniesing S, Vuijst CL, Wiersinga WM, Swaab DF. Decreased neuropeptide Y (NPY) expression in the infundibular nucleus of patients with nonthyroidal illness. Peptides. 2001;22:459-65.

38. Eledrisi MS, Verghese AC. Adrenal insufficiency in HIV infection: a review and recommendations. Am J Med Sci. 2001;321:137-44.

39. Tovo PA, de Martino M, Gabiano C, Cappello N, D'Elia R, Loy $A$, et al. Prognostic factors and survival in children with perinatal HIV infection. Lancet. 1992;339:1249-53.
40. Nachman SA, Lindsey JC, Pelton S, Mofenson L, Mclntosh K, Wiznia $A$, et al. Growth in human immunodeficiency virus-infected children receiving ritonavir-containing antiretroviral therapy. Arch Pediatr Adolesc Med. 2002;156:497-503.

41. Congote LF. Monitoring insulin-like growth factors in HIV infection and aids. Clin Chim Acta. 2005;361:30-53.

42. Meininger G, Grinspoon S. Regulation of the growth hormone/insulin-like growth factor-I axis in HIV disease. Endocrinologist. 2001;11:188-95.

43. Frost RA, Fuhrer J, Steigbigel $R$, Mariuz $P$, Lang $\mathrm{CH}$, Gelato MC. Wasting in the acquired immune deficiency syndrome is associated with multiple defects in the serum insulin-like growth factor system. Clin Endocrinol (Oxf). 1996;44:501-14.

44. Heijligenberg R, Sauerwein HP, Brabant G, Endert E, Hommes MJ, Romijn JA. Circadian growth hormone secretion in asymptomatic human immune deficiency virus infection and acquired immunodeficiency syndrome. J Clin Endocrinol Metab. 1996;81:4028-32.

45. Rondanelli M, Solerte SB, Fioravanti M, Scevola D, Locatelli $M$, Minoli $L$, et al. Circadian secretory pattern of growth hormone, insulin-like growth factor type l, cortisol, adrenocorticotropic hormone, thyroid-stimulating hormone and prolactin during HIV infection. AIDS. 1997;13:1243-9.

46. Stanley TL, Grinspoon SK. GH/GHRH axis in HIV lipodystrophy. Pituitary. 2008 Feb 13 [Epub ahead of print].

47. Viganò A, Mora S, Brambilla P, Schneider L, Merlo M, Monti LD, et al. Impaired growth hormone secretion correlates with visceral adiposity in highly active antiretroviral treated HIVinfected adolescents. AIDS. 2003;17:1435-41.

48. Van Rossum AMC, Gaaker MI, Verweel G, Hartwig NG, Wolfs TF, Geelen SP, et al. Endocrinology and immunologic factors associated with recovery of growth in children with human immunodeficiency virus type 1 infection treated with protease inhibitors. Pediatric Infect Dis J. 2003;22:70-6.

49. Schambelan M, Mulligan K, Grunfeld C, Daar ES, LaMarca A, Kotler DP, et al. Recombinant human growth hormone in patients with HIV-associated wasting. A randomized, placebocontrolled trial. Serostim Study Group. Ann Intern Med. 1996;125:873-82.

50. Krentz AJ, Koster FT, Crist DM, Finn K, Johnson LZ, Boyle PJ, et al. Anthropometric, metabolic, and immunological effects of recombinant human growth hormone in Aids and Aids-related complex. J Acquir Immune Defic Syndr. 1993;6:245-51.

51. McNurlan MA, Garlick PJ, Steigbigel RT, DeCristofaro KA, Frost RA, Lang $\mathrm{CH}$, et al. Responsiveness of muscle protein synthesis to growth hormone administration in HIV-infected individuals declines with severity of disease. J Clin Invest. 1997; 100:2125-32.

52. Waters D, Danska J, Hardy K, Koster F, Qualls C, Nickell D, et al. Recombinant human growth hormone, insulin-like growth factor 1, and combination therapy in Aids-associated wasting: a randomized, double-blind, placebocontrolled trial. Ann Intern Med. 1996;125:865-72.

53. Esposito JG, Thomas SG, Kingdon L, Ezzat S. Growth hormone treatment improves peripheral muscle oxygen extraction-utilization during exercise in patients with human immunodeficiency virus-associated wasting: A randomized controlled trial. J Clin Endocrinol Metab. 2004;89:5124-31.

54. Moyle GJ, Daar ES, Gertner JM, Kotler DP, Melchior JC, O'Brien F, et al. Serono 9037 Study Team. Growth hormone improves lean body mass, physical performance, and quality of life in subjects with HIV-associated weight loss or wasting 
on highly active antiretroviral therapy. J Acquir Immune Defic Syndr. 2004;35:367-75.

55. Storer TW, Woodhouse LJ, Sattler F, Singh AB, Schroeder ET, Beck K, et al. A randomized, placebocontrolled trial of nandrolone decanoate in human immunodeficiency virus-infected men with mild to moderate weight loss with recombinant human growth hormone as active reference treatment. J Clin Endocrinol Metab. 2005;90:4474-82.

56. Wanke C, Gerrior J, Kantaros J, Coakley E, Albrecht M. Recombinant human growth hormone improves the fat redistribution syndrome (lipodystrophy) in patients with HIV. AIDS. 1999;13:2099-103.

57. Zeitler PS, Travers S, Kappy MS. Advances in the recognition and treatment of endocrine complications in children with chronic illness. Adv Pediatr. 1999;46:101-49.
58. Napolitano LA, Schmidt D, Gotway MB, Ameli N, Filbert EL, $\mathrm{Ng} \mathrm{MM}$, et al. Growth hormone enhances thymic function in HIV-infected adults. J Clin Invest. 2008;118:1085-98.

\section{Endereço para correspondência:}

Angela Maria Spinola-Castro

Disciplina de Especialidades Pediátricas, Departamento de Pediatria, Unifesp/EPM

Rua Doutor Diogo de Faria, 307

04037-000 São Paulo, SP

E-mail: aspinola.dped@epm.br 